\title{
Strange quark content of the nucleon and dark matter searches
}

\section{R. D. Young*}

Special Research Centre for the Subatomic Structure of Matter (CSSM) and ARC Centre of Excellence in Particle Physics at the Terascale (CoEPP), School of Chemistry and Physics, University of Adelaide, SA 5005, Australia.

E-mail: ross.young@adelaide.edu.au

The strange quark scalar content plays an important role in both the description of nucleon structure and in the determination of dark matter direct detection cross sections. As a measure of the strange-quark contribution to the nucleon mass, the strange-quark sigma term $\left(\sigma_{s}\right)$ provides important insight into the nature of mass generation in QCD. The phenomenological determination of $\sigma_{s}$ exhibits a wide range of variation, with values suggesting that the strange quark contributes anywhere between 0 and more than $30 \%$ of the nucleon mass. In the context of dark matter searches, coupled with relatively large Higgs coupling to strangeness, this variation dominates the uncertainty in predicted cross sections for a large class of dark matter models. Here we report on the recent results in lattice QCD, which are now giving a far more precise determination of $\sigma_{s}$ than can be inferred from phenomenology. As a consequence, the lattice determinations of $\sigma_{s}$ can now dramatically reduce the uncertainty in dark matter cross sections associated with the hadronic matrix elements.

The 30 International Symposium on Lattice Field Theory - Lattice 2012,

June 24-29, 2012

Cairns, Australia

${ }^{*}$ Speaker. 


\section{Introduction}

The modern picture of the universe suggests that the ordinary matter component in the energy composition of the universe is only about $4 \%$ - with the remainder comprised of some form of cold, "dark matter", which clusters around ordinary matter and an even larger "dark energy" component. While little is known about the physical nature of dark energy, there is strong evidence that suggests we are nearing the discovery phase in the identification of dark matter. Supposing dark matter to take a particle-like form, the relevant mass scale for such particles are most likely to be within reach of the LHC.

For a general spin-independent interaction of a WIMP with a nucleus, the low-energy limit reduces to a scalar contact interaction, and hence sensitive to the $\bar{q} q$ matrix elements within a nucleon. And for Higgs-dominated exchange, these couplings are proportional to the corresponding quark mass and are hence sensitive to the nucleon sigma terms. The sigma terms therefore become of principal uncertainty in the predicting the cross sections associated with any candidate model of dark matter. The significance of the uncertainty in these hadronic matrix elements has been highlighted in a range of dark matter models, see for example Refs. [1, 2, 3, 4, 5].

Before going on to discuss the sigma terms, and the strangeness scalar content, it is worth recapping some recent advances in resolving the strange quark vector form factors in the nucleon. Early estimates of the strangeness electromagnetic currents had suggested that they could be relatively large [6]. After a dedicated experimental effort, parity-violating electron scattering measurements $[7,8,9]$ have revealed that the strange quarks contribute much less than originally suggested. This finding is also supported by lattice-based phenomenological estimates $[10,11]$ and recent direct lattice QCD simulation results $[12,25]$. For a recent review of these latest revelations, see Ref. [13].

After setting some general notation, the phenomenological determination of the sigma terms are reviewed in Section 2; a summary of the latest lattice QCD results are reported in Section 3; the significance of these results in the context of dark matter searches are discussed in Section 4; follwed with a summary in Section 5 .

\subsection{Notation}

Of primary interest here are the scalar nucleon matrix elements, where we'll use the notation for the light- and strange-quark matrix elements

$$
\sigma_{l} \equiv m_{l}\langle N|\bar{u} u+\bar{d} d| N\rangle, \quad \sigma_{s} \equiv m_{s}\langle N|\bar{s} s| N\rangle,
$$

with the average light-quark mass given by $m_{l} \equiv\left(m_{u}+m_{d}\right) / 2$.

A commonly reported measure of the strangeness sigma term is through the $y$-parameter,

$$
y \equiv \frac{2\langle N|\bar{s} s| N\rangle}{\langle N|\bar{u} u+\bar{d} d| N\rangle}=\frac{2 m_{l}}{m_{s}} \frac{\sigma_{s}}{\sigma_{l}} .
$$

\section{Phenomenological Determination}

By the very nature of the weak coupling of the Higgs to the low-energy sector of the Standard Model, the sigma terms are essentially impossible to measure directly. Fortunately, $\sigma_{l}$ can be inferred through a chiral low-energy relation, where the amount of explicit chiral symmetry breaking 
can be related to pion-nucleon scattering. In particular, the light-quark sigma term can be extracted through measurement of the Born-subtracted, isoscalar amplitude $\Sigma_{\pi N}(t)$.

Both $\Sigma_{\pi N}$ and $\sigma$ vanish with the quark mass, but importantly they become equal as the chiral limit is approached. That is, the leading dependence of the quark mass is the same, with the leading difference being $\mathscr{O}\left(\mathrm{m}_{l}^{3 / 2}\right)$. The limited knowledge of this difference term can further be reduced by moving to the unphysical kinematic point $t=2 m_{\pi}^{2}$ (the Cheng-Dashen point [14]), where the remainder is $\mathscr{O}\left(m_{l}^{2}\right)$ [15], being defined by

$$
\Delta_{R} \equiv \Sigma_{\pi N}\left(t=2 m_{\pi}^{2}\right)-\sigma_{l}\left(t=2 m_{\pi}^{2}\right)=\mathscr{O}\left(m_{l}^{2}\right) .
$$

Here, the scalar matrix element has been extended to non-zero momentum transfer, with the usual sigma term corresponding to the $t \rightarrow 0$ limit, $\sigma_{l}=\sigma_{l}(t=0)$. An early calculation of the remainder term has determined an estimate $\Delta_{R} \simeq 0.35 \mathrm{MeV}$ [16], later followed by an updated value $\Delta_{R} \simeq$ $2 \mathrm{MeV}$ [17].

Extraction of the sigma term at $t=0$ then requires the determination of the form factor correction, defined by

$$
\Delta_{\sigma} \equiv \sigma_{l}\left(t=2 m_{\pi}^{2}\right)-\sigma_{l}(t=0) .
$$

Through dispersion relations, this form factor correction has been estimated to be $\Delta_{\sigma} \simeq 15 \mathrm{MeV}$ [18].

With the required theoretical corrections under reasonable control, the pion-nucleon sigma term can then be extracted from the experimentally determined $\Sigma_{\pi N}$, as extrapolated to the unphysical Cheng-Dashen point. In summary, $\sigma_{l}$ is determined by

$$
\sigma_{l}=\Sigma_{\pi N}\left(t=2 m_{\pi}^{2}\right)-\Delta_{R}-\Delta_{\sigma}
$$

Following this outlined technique, analysis of $\pi N$ scattering data gives the Gasser-LeutwylerSainio (GLS) value $\sigma_{l}=45 \pm 8 \mathrm{MeV}$ [16], or a somewhat larger value from the George Washington University/TRIUMF (GWU) group analysis $\sigma_{l}=64 \pm 7 \mathrm{MeV}$ [19].

In addition to these benchmark calculations of GLS and GWU, an analysis based on a covariant baryon chiral perturbation theory has recently been reported by Alarcón, Martin-Camalich \& Oller (AMO) [20]. Here the low-energy $\pi N$ scattering phase shifts are fit directly within the effective field theory framework. The analysis determines the relevant low-energy constants necessary for the extraction of the sigma term by the Hellmann-Feynman theorem (discussed below). Together with estimates for the higher-order terms, the sigma term is reported to be $\sigma_{l}=59 \pm 7 \mathrm{MeV}$, lying between the two values reported above.

Estimating the strange-quark sigma term is significantly more challenging. In this case, the strange quark is too heavy to reliably truncate the low-energy relation at low order. As an alternative, the conventional approach has been to estimate $\sigma_{s}$ by studying the $\mathrm{SU}(3)$ breaking among the baryon octet [21, 22]. Here, the baryon mass splittings can be used to constrain the non-singlet combination

$$
\sigma_{0} \equiv m_{l}\langle N|\bar{u} u+\bar{d} d-2 \bar{s} s| N\rangle .
$$

To leading-order in the quark masses, $\sigma_{0}$ can be estimated from the physically observed spectrum by

$$
\sigma_{0} \simeq \frac{m_{l}}{m_{s}-m_{l}}\left(M_{\Sigma}+M_{\Xi}-2 M_{N}\right) \simeq 24 \mathrm{MeV} .
$$




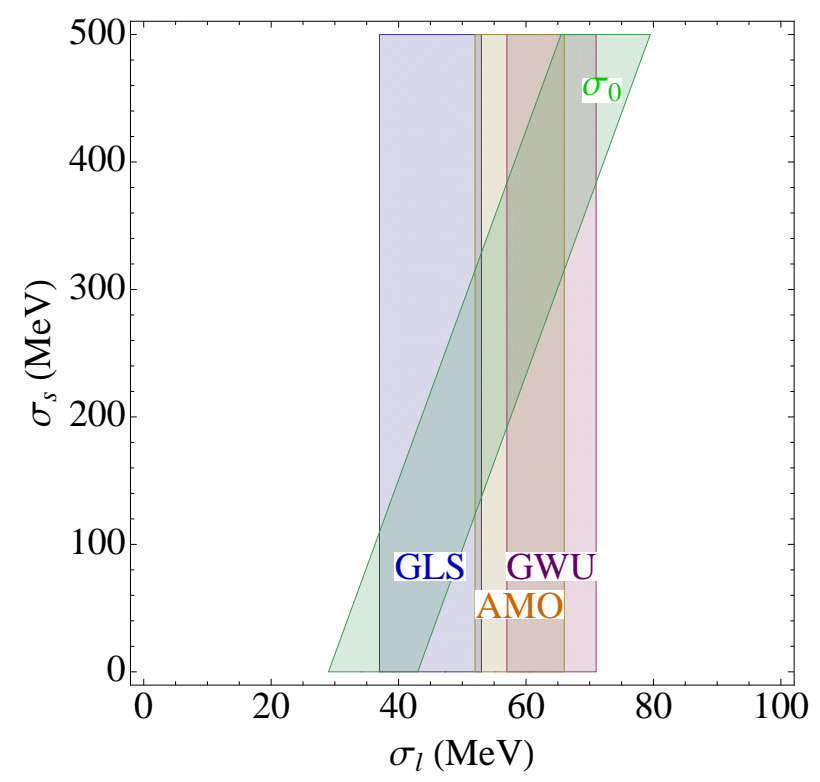

Figure 1: The determination of $\sigma_{s}$, from the best-estimate of $\sigma_{0}=36 \pm 7 \mathrm{MeV}$ [22], has a high degree of sensitivity to the determination of pion-nucleon sigma term. The three values for $\sigma_{l}$ are as discussed in the text.

By incorporating the higher-order terms in the quark mass expansion, Borasoy and Meißner have determined an improved estimate $\sigma_{0}=36 \pm 7 \mathrm{MeV}$ [22]. With an estimate for $\sigma_{0}$, the strangeness sigma term is then given by

$$
\sigma_{s}=\frac{m_{s}}{2 m_{l}}\left(\sigma_{l}-\sigma_{0}\right) .
$$

Being multiplied by the large quark mass ratio, this method leads to a value for $\sigma_{s}$ that is acutely sensitive to the difference between $\sigma_{l}$ and $\sigma_{0}$. Further, given the limited precision available to $\sigma_{0}$, even a perfect determination of $\sigma_{l}$ leaves a residual uncertainty in $\sigma_{s}$ of order $90 \mathrm{MeV}$. For the three determinations of $\sigma_{l}$ discussed above, Figure 1 displays the broad range of possible $\sigma_{s}$ values.

Given the difficulty in extracting a precise determination of $\sigma_{s}$ from phenomenology, there is significant scope for lattice QCD to provide a meaningful constraint on this nucleon matrix element. In addition, there is also the potential for lattice simulations to shed light on the phenomenological determination of $\sigma_{l}$.

\section{Lattice QCD}

Within the framework of lattice QCD, there are two main methods used in the extraction of the sigma terms. These divide into the explicit evaluation of the scalar $\bar{q} q$ matrix element by 3point function methods or by invoking the Hellmann-Feynman relation through the study of the quark-mass dependence of the nucleon mass.

In the first technique, appropriate ratios of 3-point and 2-point correlation functions are formed to isolate the matrix elements of interest. The evaluation of the correlation functions involves two distinct forms of Wick contraction, as depicted in Figure 2. In particular, there are contributions 

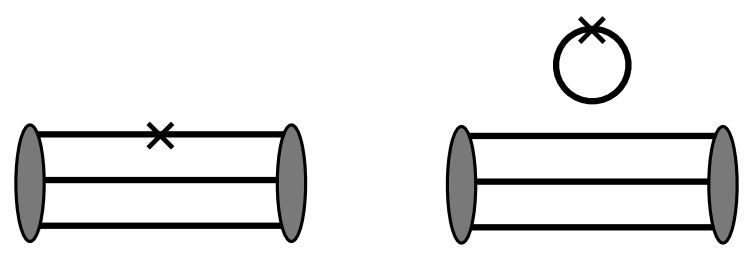

Figure 2: Schematic diagram displaying the two topologically distinct contractions in the evaluation of the scalar 3-point function. The standard jargon is that the left is a connected insertion and the right a disconnected insertion.

involving quark line connected or disconnected operator insertions. While standard methods typically yield strong signals for the connected correlation functions, it is well-known that the disconnected insertions are notoriously challenging, see eg. Refs. [23, 24, 25]. This of particular significance for the strange-quark matrix elements in the nucleon, which are purely disconnected.

A common alternative to the three-point method, is to determine the $\bar{q} q$ matrix elements by differentiation with respect to the quark masses, where the Hellmann-Feynman relation gives [26, $27,28]$

$$
\sigma_{q}=m_{q} \frac{\partial M_{N}}{\partial m_{q}} .
$$

Here the Gell-Mann-Oakes-Renner (GOR) relation [29] is commonly imposed such that $M_{N}$ is expressed as a function of the squares of meson masses. One way to do this is to write $M_{N}=$ $M_{N}\left(m_{\pi}^{2}, \tilde{m}_{K}^{2}\right)$, where $\tilde{m}_{K}^{2}=m_{K}^{2}-m_{\pi}^{2} / 2$ is the projection of the square of the kaon mass onto the $\mathrm{SU}(2)$ chiral limit $\left(m_{l} \rightarrow 0\right)$. With such a formulation, the sigma terms (for $2+1$-flavour simulations) are easily written as

$$
\sigma_{l}=m_{\pi}^{2} \frac{\partial M_{N}}{\partial m_{\pi}^{2}}, \quad \sigma_{s}=\tilde{m}_{K}^{2} \frac{\partial M_{N}}{\partial \tilde{m}_{K}^{2}} .
$$

The main challenge of this approach is the difficulty in reliably parameterising the quark-mass dependence over a range of light and strange quark masses. In particular, it is only in recent years that there have been large scale numerical simulations of baryons with 2+1-flavours of dynamical quarks, eg. [30, 31, 32, 33, 34, 35, 36]. In constraining the two-dimensional parameter space, it is also the case that typical lattice trajectories in the $m_{l}-m_{s}$ plane approach the physical point for approximately constant $m_{s}$; though the QCDSF-UKQCD are currently pursuing an approach which keeps the singlet combination $\left(2 m_{l}+m_{s}\right)$ a constant [36].

A summary of the progression of lattice results is displayed in Figures 3 and 4 . With differing degrees of analysis into the various uncertainties of the calculations, it would be difficult to formulate any rigourous aggregates. Nevertheless, two clear features are emergent. For $\sigma_{l}$, the values revealed in lattice simulations are compatible with the range of phenomenologically determined values. Secondly, the modern values of $\sigma_{s}$ are all at the lower end of the possible values suggested by Figure 1. To highlight the current status, Figure 5 shows a close-up of the recent determinations of $\sigma_{s}$. 


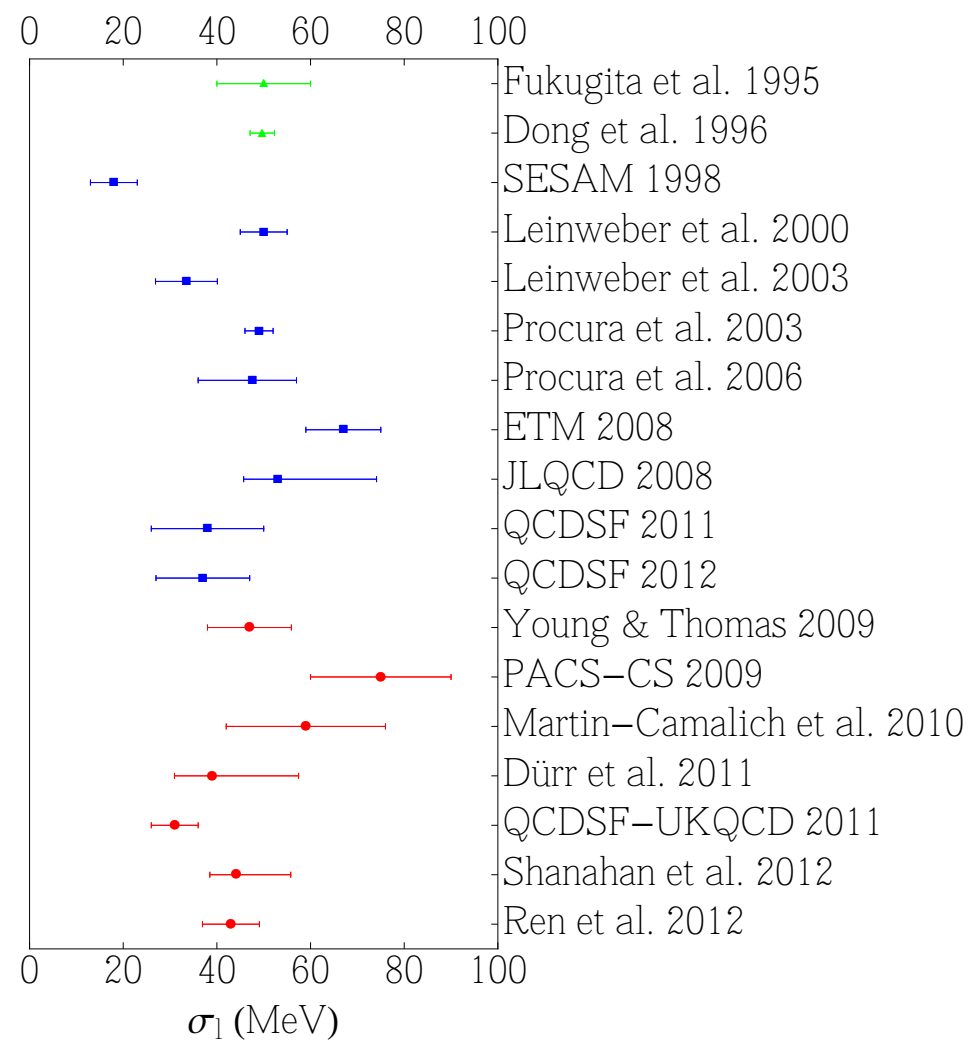

Figure 3: Light-quark sigma term results based on lattice QCD. The colours denoted the number of dynamical flavours of quarks: green is $N_{f}=0$, blue $N_{f}=2$ and red $N_{f} \geq 2+1$. References: Fukugita $e t$ al. [37], Dong et al. [38], SESAM [39], Leinweber et al. (2000) [40], Leinweber et al. (2003) [41], Procura et al. (2003) [42], Procura et al. (2006) [43], ETM [44], JLQCD [45], QCDSF (2011) [46], QCDSF (2012) [47], Young \& Thomas [48], PACS-CS [49], Martin-Camalich et al. [50], Dürr et al. [51], QCDSF-UKQCD [52], Shanahan et al. [53], Ren et al. [54].

\section{Dark Matter}

The smaller values of $\sigma_{s}$ revealed in the recent lattice studies are particularly significant in the context of the direct search for dark matter. The most precise limits on WIMP-nucleon cross sections are being constrained by the XENON100 Collaboration, with the latest update placing an upper bound on the cross section of less than $10^{-44} \mathrm{~cm}^{2}$ over a wide range of WIMP masses [61]. Figure 3 of [61] suggests these limits are continuing to reduce the parameter space of potential supersymmetric candidates for dark matter.

The XENON100 Collaboration results are plotted against predicted cross sections for some favoured supersymmetric models $[62,63,64]$. The predicted cross section rates are based on a determination of the strange quark sigma term, $\sigma_{s}{ }^{1}$ as outlined in Section 2. Hence $\sigma_{s}$ in these studies exhibits the extreme sensitivity to $\sigma_{l}$ displayed in Figure 1.

As the WIMP-nucleon interactions are largely Higgs-coupling driven, the difference between a small and large $\sigma_{s}$ can have a dramatic influence on the predicted cross sections. This is high-

\footnotetext{
${ }^{1}$ Or in an alternative common notation, $f_{T s}=\sigma_{s} / M_{p}$, for the proton mass $M_{p}$.
} 


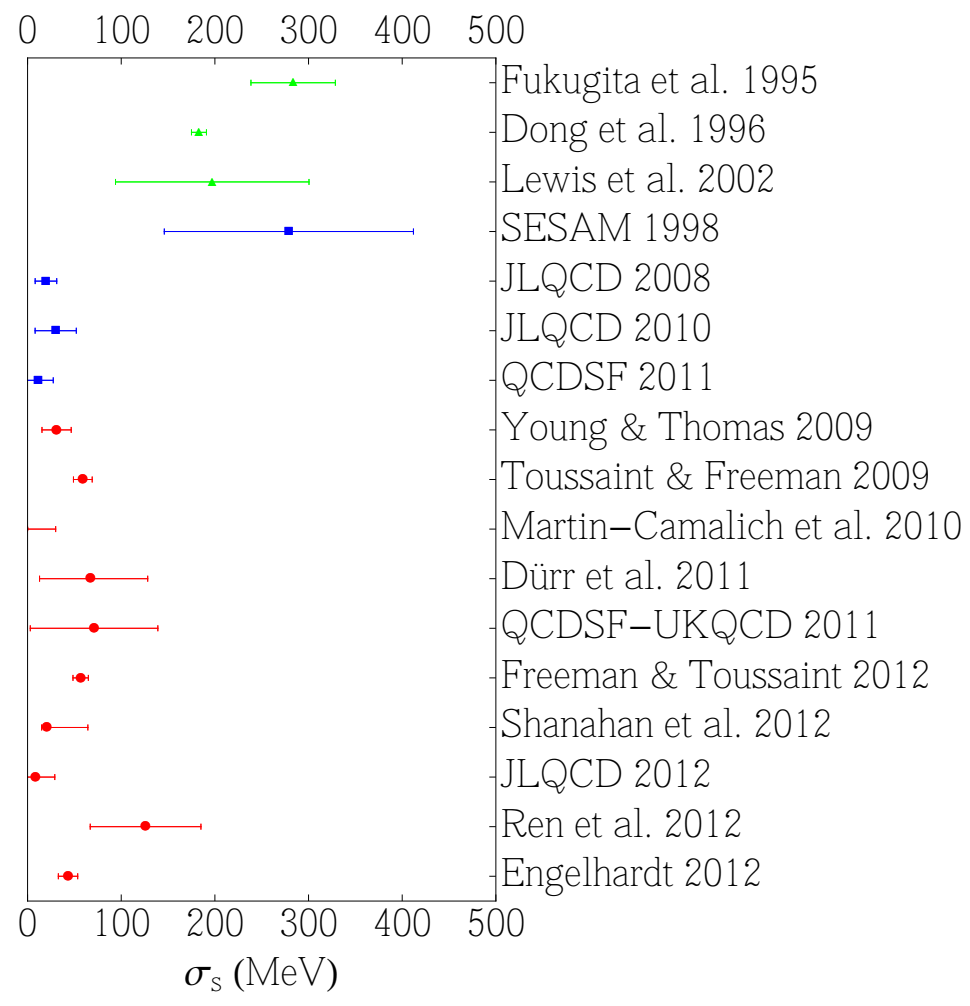

Figure 4: Strange-quark sigma term results based on lattice QCD. Colours as in Figure 3. Fukugita et al. [37], Dong et al. [38], Lewis et al. [55], SESAM [39], JLQCD (2008) [45], JLQCD 2010 [56], QCDSF [46], Young \& Thomas [48], Toussaint \& Freeman [57], Martin-Camalich et al. [50], Dürr et al. [51], QCDSF-UKQCD [52], Freeman \& Toussaint [58], Shanahan et al. [53], JLQCD (2012) [59], Ren et al. [54], Engelhardt [60].

lighted in Figure 6, which shows how the predicted cross section for a particular constrained minimal supersymmetric standard model (CMSSM) model ${ }^{2}$ depends strongly on $\Sigma_{\pi N}$ (with $\sigma_{s}$ constrained by the phenomenological $\sigma_{0}$ ) [65]. In contrast, the displayed ellipse shows the range of predicted cross sections within the 95\% confidence level interval of the lattice QCD determinations of $\sigma_{l}$ and $\sigma_{s}$ from Refs. [48, 57]. It should be stressed that the reduced variation in the cross section is a consequence of the increased precision in $\sigma_{s}$ from lattice QCD input - which is not reliant on the propogation of the phenomenological uncertainty in $\sigma_{0}$.

Generic dark matter cross section packages, such as micrOMEGAs [68], have been designed to take as inputs $\sigma_{l}$ and $\sigma_{0}$. With the improvement in lattice QCD results discussed above, it would be advantageous to see these packages reformulated to take $\sigma_{l}$ and $\sigma_{s}$ as inputs ${ }^{3}$. In the meantime, with cross section predictions based on $\sigma_{0}$ as an input, the reduction in uncertainty in $\sigma_{s}$ may be equivalently stated as a reduction in $\sigma_{l}-\sigma_{0}$, cf. Eq. (2.6). A crude, yet conservative view of

\footnotetext{
${ }^{2}$ The figure displays the predicted cross-section for "model C", as one of a class of benchmark models proposed pre-LHC $[66,67]$.

${ }^{3}$ Of course these are precisely the same thing with an appropriately included correlation coefficient.
} 


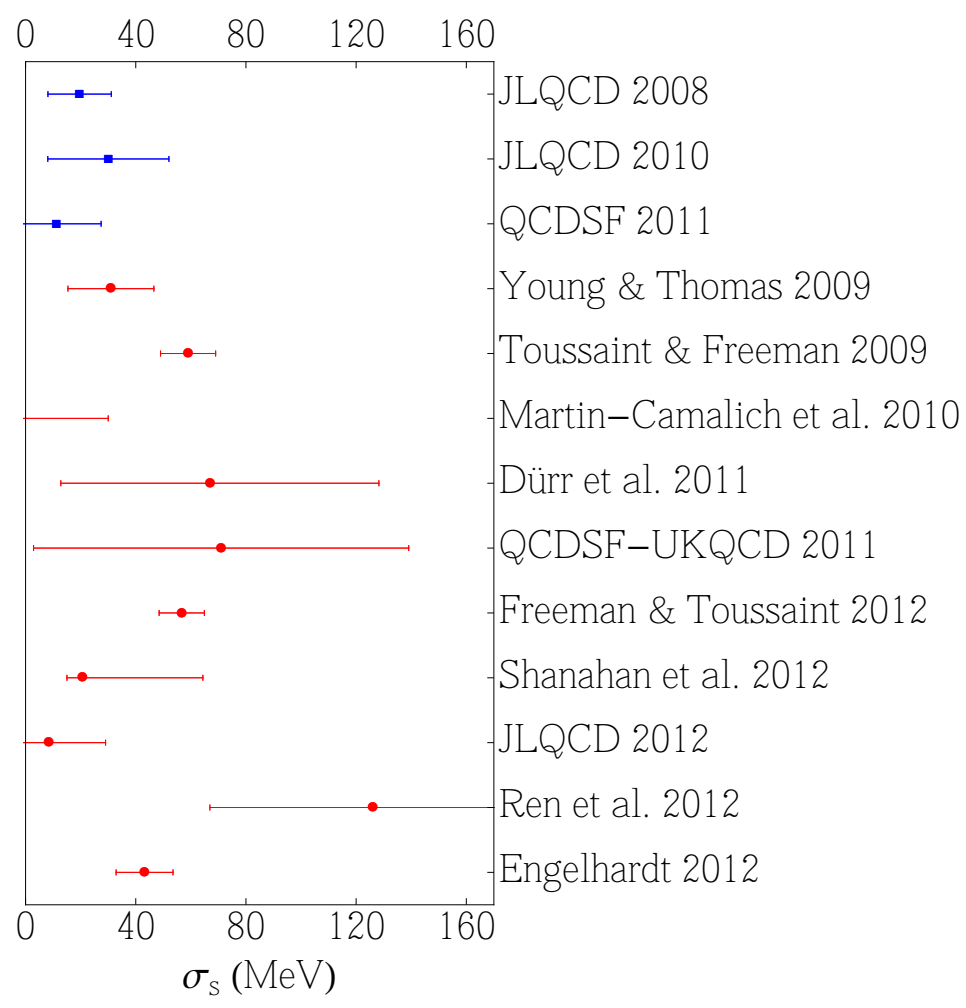

Figure 5: Zoomed in graphic of Fig. 2 showing more recent results on $\sigma_{s}$.

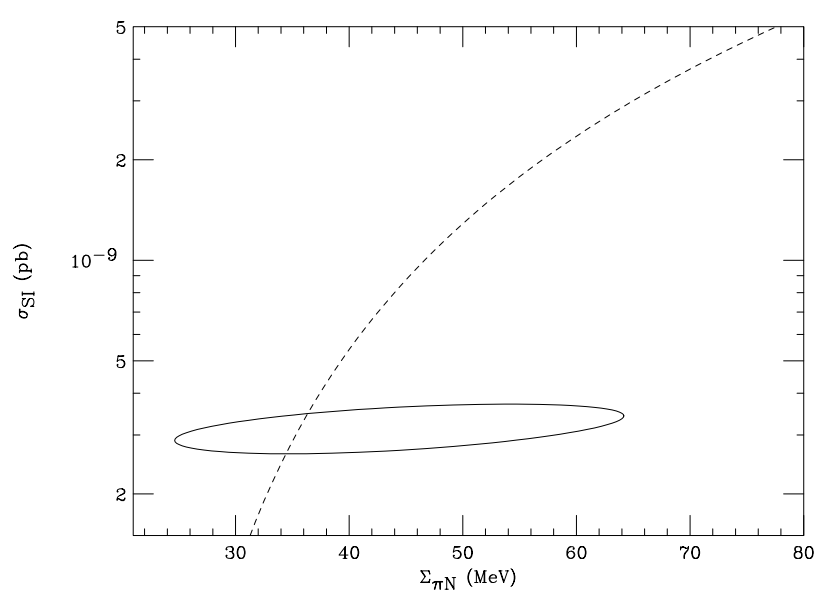

Figure 6: For a pre-LHC CMSSM dark matter model, the predicted spin-independent cross section $\left(\sigma_{\mathrm{SI}}\right)$ shows a strong dependence on $\Sigma_{\pi N}$. This variation is a consequence of the large variation of $\sigma_{s}$, as constrained by the phenomenological $\sigma_{0}$, as seen in Figure 1. The ellipse show the range of $\sigma_{\mathrm{SI}}$ using lattice inputs for $\Sigma_{\pi N}$ and $\sigma_{s}$ [65]. 
Figure 5, may suggest a value ${ }^{4}$

$$
\sigma_{l}-\sigma_{0}=\frac{2 m_{l}}{m_{s}}(40 \pm 30 \mathrm{MeV}) \simeq 2.9 \pm 2.2 \mathrm{MeV} .
$$

Already at this scale of precision, there should be a substantial reduction in the uncertainties in $\sigma_{\mathrm{SI}}$ associated with the hadronic matrix elements. Importantly, with the reduction in the hadronic uncertainty, any discovery of dark matter will have significantly more discrimination power among candidate models.

\section{Summary}

To summarise, an accurate determination of the strange quark sigma term is of principle importance in the reduction of hadronic uncertainties in the predicted dark matter cross sections for a wide range of models. In the determination of the relevant nucleon scalar matrix elements, lattice QCD simulations have made significant progress in recent years - particularly with the emergence of dynamical simulations with $2+1$ flavours of dynamical quarks. As a confirmation of lattice methods, it is reassuring to observe that the pion-nucleon sigma term appears to be compatible with the reliably determined phenomenological extraction. Further, recent determinations of the strange quark sigma term are significantly smaller than had previously been suggested - and lattice calculations are now at a far greater precision. With the prospect of a discovery of dark matter in the near future, it will be essential for lattice QCD simulations to further reduce these hadronic uncertainties.

\section{Acknowledgements}

I thank my collaborators for their contributions to various aspects of the work presented here, J. Giedt, P.E. Shanahan, A.W. Thomas and S.J. Underwood. This work was supported by the Australian Research Council through the ARC Centre of Excellence for Particle Physics at the Terascale and grants DP110101265 and FT120100821.

\section{References}

[1] A. Bottino, F. Donato, N. Fornengo and S. Scopel, Astropart. Phys. 13, 215 (2000) [hep-ph/9909228].

[2] J. R. Ellis, K. A. Olive, Y. Santoso and V. C. Spanos, Phys. Rev. D 71, 095007 (2005) [hep-ph/0502001].

[3] J. R. Ellis et al., Phys. Rev. D 77, 065026 (2008) [arXiv:0801.3656 [hep-ph]].

[4] A. Bottino, F. Donato, N. Fornengo and S. Scopel, Phys. Rev. D 78, 083520 (2008) [arXiv:0806.4099 [hep-ph]].

[5] R. J. Hill and M. P. Solon, Phys. Lett. B 707, 539 (2012) [arXiv:1111.0016 [hep-ph]].

[6] R. L. Jaffe, Phys. Lett. B 229, 275 (1989).

\footnotetext{
${ }^{4}$ The final numerical value uses the central estimate of the quark mass ratio from the FLAG [69], which is primarily determined by the precision studies of Refs. [70, 71].
} 
[7] F. E. Maas et al., Phys. Rev. Lett. 94, 152001 (2005) [nucl-ex/0412030].

[8] D. S. Armstrong et al. [G0 Collaboration], Phys. Rev. Lett. 95, 092001 (2005) [nucl-ex/0506021].

[9] A. Acha et al. [HAPPEX Collaboration], Phys. Rev. Lett. 98, 032301 (2007) [nucl-ex/0609002].

[10] D. B. Leinweber et al., Phys. Rev. Lett. 94, 212001 (2005) [hep-lat/0406002].

[11] D. B. Leinweber et al., Phys. Rev. Lett. 97, 022001 (2006) [hep-lat/0601025].

[12] T. Doi et al., Phys. Rev. D 80, 094503 (2009) [arXiv:0903.3232 [hep-ph]].

[13] K. Paschke, A. Thomas, R. Michaels and D. Armstrong, J. Phys. Conf. Ser. 299, 012003 (2011).

[14] T. P. Cheng and R. F. Dashen, Phys. Rev. Lett. 26, 594 (1971).

[15] L. S. Brown, W. J. Pardee and R. D. Peccei, Phys. Rev. D 4, 2801 (1971).

[16] J. Gasser, H. Leutwyler and M. E. Sainio, Phys. Lett. B 253, 252 (1991).

[17] V. Bernard et al., Phys. Lett. B 389, 144 (1996) [arXiv:hep-ph/9607245].

[18] J. Gasser, H. Leutwyler and M. E. Sainio, Phys. Lett. B 253, 260 (1991).

[19] M. M. Pavan et al., PiN Newslett. 16, 110 (2002) [arXiv:hep-ph/0111066].

[20] J. M. Alarcon, J. Martin Camalich and J. A. Oller, Phys. Rev. D 85, 051503 (2012) [arXiv:1110.3797 [hep-ph]].

[21] J. Gasser, Annals Phys. 136, 62 (1981).

[22] B. Borasoy and U.-G. Meißner, Annals Phys. 254, 192 (1997) [arXiv:hep-ph/9607432].

[23] G. S. Bali, S. Collins and A. Schafer, Comput. Phys. Commun. 181, 1570 (2010) [arXiv:0910.3970 [hep-lat]].

[24] S. Dinter et al. [ETM Collaboration], JHEP 1208, 037 (2012) [arXiv:1202.1480 [hep-lat]].

[25] R. Babich et al., Phys. Rev. D 85, 054510 (2012) [arXiv:1012.0562 [hep-lat]].

[26] H. Hellmann, Einführung in die Quantenchemie (Deuticke Verlag, Leipzig, 1937).

[27] R. P. Feynman, Phys. Rev. 56, 340 (1939).

[28] J. Gasser and A. Zepeda, Nucl. Phys. B 174, 445 (1980).

[29] M. Gell-Mann, R. J. Oakes and B. Renner, Phys. Rev. 175, 2195 (1968).

[30] C. W. Bernard et al., Phys. Rev. D 64, 054506 (2001) [arXiv:hep-lat/0104002].

[31] A. Walker-Loud et al. [LHPC], Phys. Rev. D 79, 054502 (2009) [arXiv:0806.4549 [hep-lat]].

[32] S. Aoki et al. [PACS-CS], Phys. Rev. D 79, 034503 (2009) [arXiv:0807.1661 [hep-lat]].

[33] H. W. Lin et al. [HSC], Phys. Rev. D 79, 034502 (2009) [arXiv:0810.3588 [hep-lat]].

[34] S. Dürr et al., Science 322, 1224 (2008) [arXiv:0906.3599 [hep-lat]].

[35] R. Baron et al. [ETM Collaboration], JHEP 1006, 111 (2010) [arXiv:1004.5284 [hep-lat]].

[36] W. Bietenholz et al., Phys. Rev. D 84, 054509 (2011) [arXiv:1102.5300 [hep-lat]].

[37] M. Fukugita et al., Phys. Rev. D 51, 5319 (1995) [arXiv:hep-lat/9408002].

[38] S. J. Dong et al., Phys. Rev. D 54, 5496 (1996) [arXiv:hep-ph/9602259]. 
[39] S. Güsken et al. [SESAM], Phys. Rev. D 59, 054504 (1999) [arXiv:hep-lat/9809066].

[40] D. B. Leinweber et el., Phys. Lett. B 482, 109 (2000) [arXiv:hep-lat/0001007].

[41] D. B. Leinweber et al., Phys. Rev. Lett. 92, 242002 (2004) [arXiv:hep-lat/0302020].

[42] M. Procura et al., Phys. Rev. D 69, 034505 (2004) [arXiv:hep-lat/0309020].

[43] M. Procura et al., Phys. Rev. D 73, 114510 (2006) [arXiv:hep-lat/0603001].

[44] C. Alexandrou et al. [ETM], Phys. Rev. D 78, 014509 (2008) [arXiv:0803.3190 [hep-lat]].

[45] H. Ohki et al. [JLQCD], Phys. Rev. D 78, 054502 (2008) [arXiv:0806.4744 [hep-lat]].

[46] G. S. Bali et al. [QCDSF Collaboration], Phys. Rev. D 85, 054502 (2012) [arXiv:1111.1600 [hep-lat]].

[47] G. S. Bali et al. [QCDSF Collaboration], Nucl. Phys. B 866, 1 (2013) [arXiv:1206.7034 [hep-lat]].

[48] R. D. Young and A. W. Thomas, Phys. Rev. D 81, 014503 (2010) [arXiv:0901.3310 [hep-lat]].

[49] K. I. Ishikawa et al. [PACS-CS], Phys. Rev. D 80, 054502 (2009) [arXiv:0905.0962 [hep-lat]].

[50] J. Martin Camalich, L. S. Geng and M. J. Vicente Vacas, Phys. Rev. D 82, 074504 (2010) [arXiv:1003.1929 [hep-lat]].

[51] S. Dürr et al., Phys. Rev. D 85, 014509 (2012) [arXiv:1109.4265 [hep-lat]].

[52] R. Horsley et al., Phys. Rev. D 85, 034506 (2012) [arXiv:1110.4971 [hep-lat]].

[53] P. E. Shanahan, A. W. Thomas and R. D. Young, arXiv:1205.5365 [nucl-th].

[54] X. -L. Ren, L. S. Geng, J. M. Camalich, J. Meng and H. Toki, arXiv:1209.3641 [nucl-th].

[55] R. Lewis, W. Wilcox and R. M. Woloshyn, Phys. Rev. D 67, 013003 (2003) [hep-ph/0210064].

[56] K. Takeda et al. [JLQCD Collaboration], Phys. Rev. D 83, 114506 (2011) [arXiv:1011.1964 [hep-lat]].

[57] D. Toussaint and W. Freeman [MILC], Phys. Rev. Lett. 103, 122002 (2009) [arXiv:0905.2432 [hep-lat]].

[58] W. Freeman et al. [MILC Collaboration], arXiv:1204.3866 [hep-lat].

[59] H. Ohki et al. [JLQCD Collaboration], arXiv:1208.4185 [hep-lat].

[60] M. Engelhardt, arXiv:1210.0025 [hep-lat].

[61] E. Aprile et al. [XENON100 Collaboration], Phys. Rev. Lett. 109, 181301 (2012) [arXiv:1207.5988 [astro-ph.CO]].

[62] C. Strege et al., JCAP 1203, 030 (2012) [arXiv:1112.4192 [hep-ph]].

[63] A. Fowlie et al., Phys. Rev. D 86, 075010 (2012) [arXiv:1206.0264 [hep-ph]].

[64] O. Buchmueller et al., Eur. Phys. J. C 72, 2020 (2012) [arXiv:1112.3564 [hep-ph]].

[65] J. Giedt, A. W. Thomas and R. D. Young, Phys. Rev. Lett. 103, 201802 (2009) [arXiv:0907.4177 [hep-ph]].

[66] M. Battaglia, A. De Roeck, J. R. Ellis, F. Gianotti, K. T. Matchev, K. A. Olive, L. Pape and G. Wilson, Eur. Phys. J. C 22, 535 (2001) [hep-ph/0106204].

[67] M. Battaglia, A. De Roeck, J. R. Ellis, F. Gianotti, K. A. Olive and L. Pape, Eur. Phys. J. C 33, 273 (2004) [hep-ph/0306219]. 
[68] G. Belanger, F. Boudjema, A. Pukhov and A. Semenov, Comput. Phys. Commun. 180, 747 (2009) [arXiv:0803.2360 [hep-ph]].

[69] G. Colangelo, S. Durr, A. Juttner, L. Lellouch, H. Leutwyler, V. Lubicz, S. Necco and C. T. Sachrajda et al., Eur. Phys. J. C 71, 1695 (2011) [arXiv:1011.4408 [hep-lat]].

[70] A. Bazavov et al., Rev. Mod. Phys. 82, 1349 (2010) [arXiv:0903.3598 [hep-lat]].

[71] S. Dürr et al., Phys. Lett. B 701, 265 (2011) [arXiv:1011.2403 [hep-lat]]. 